Marquette University

e-Publications@Marquette

College of Nursing Faculty Research and

Publications

Nursing, College of

$10-1-2013$

\title{
Use of Urinary Pregnanediol 3-glucuronide to Confirm Ovulation
}

R. Ecochard

Hospices Civils de Lyon, Service de Biostatistique

R. Leiva

Bruyère Research Institute

Thomas Bouchard

University of Calgary

H. Boehringer

Diagnostic Consulting Network

A. Direito

Hospices Civils de Lyon, Service de Biostatistique

See next page for additional authors

Accepted version. Steroids, Vol. 78, No. 10 (October 2013): 1035-1040. DOI. (C) 2013 Elsevier B.V. Used with permission. 
Authors

R. Ecochard, R. Leiva, Thomas Bouchard, H. Boehringer, A. Direito, A. Mariani, and Richard Fehring 


\section{Marquette University \\ e-Publications@Marquette}

\section{Nursing Faculty Research and Publications/College of Nursing}

This paper is NOT THE PUBLISHED VERSION; but the author's final, peer-reviewed manuscript. The published version may be accessed by following the link in the citation

below.

Steroids, Vol. 78, No. 10 (2013): 1035-1040. DOI. This article is (c Elsevier and permission has been granted for this version to appear in e-Publications@Marquette. Elsevier does not grant permission for this article to be further copied/distributed or hosted elsewhere without the express permission from Elsevier.

\section{Use of Urinary Pregnanediol 3- glucuronide to Confirm Ovulation}

R. Ecochard

Hospices Civils de Lyon, Service de Biostatistique, Lyon, France

Université de Lyon, Lyon, France

Université Lyon 1, Villeurbanne, France

CNRS, UMR5558, Laboratoire de Biométrie et Biologie Evolutive, Equipe Biostatistique-Santé, Villeurbanne, France

R. Leiva

Bruyère Research Institute, CT Lamont Primary Health Care Research Centre, Ottawa,

Canada

University of Ottawa, Department of Family Medicine, Ottawa, Canada

T. Bouchard

Department of Family Medicine, University of Calgary, Calgary, Alberta, Canada

H. Boehringer

Diagnostic Consulting Network, Carlsbad, CA 


\section{A. Direito}

Hospices Civils de Lyon, Service de Biostatistique, Lyon, France

Université de Lyon, Lyon, France

Université Lyon, Villeurbanne, France

Laboratoire de Biométrie et Biologie Evolutive, Equipe Biostatistique-Santé, France

A. Mariani

Service de Pédiatrie, Centre hospitalier Bassin de Thau, France

R. Fehring

College of Nursing, Marquette University, Milwaukee, WI

\section{Abstract \\ Objective}

Urinary hormonal markers may assist in increasing the efficacy of Fertility Awareness Based Methods (FABM). This study uses urinary pregnanediol-3a-glucuronide (PDG) testing to more accurately identify the infertile phase of the menstrual cycle in the setting of FABM.

\section{Methods}

Secondary analysis of an observational and simulation study, multicentre, European study. The study includes 107 women and tracks daily first morning urine (FMU), observed the changes in cervical mucus discharge, and ultrasonography to identify the day of ovulation over 326 menstrual cycles. The following three scenarios were tested: (A) use of the daily pregnandiol-3a-glucuronide (PDG) test alone; (B) use of the PDG test after the first positive urine luteinizing hormone (LH) kit result; (C) use of the PDG test after the disappearance of fertile type mucus. Two models were used: (1) one day of PDG positivity; or (2) waiting for three days of PDG positivity before declaring infertility.

\section{Results}

After the first positivity of a $\mathrm{LH}$ test or the end of fertile mucus, three consecutive days of PDG testing over a threshold of $5 \mu \mathrm{g} / \mathrm{mL}$ resulted in a $100 \%$ specificity for ovulation confirmation. They were respectively associated an identification of an average of 6.1 and 7.6 recognized infertile days.

\section{Conclusions}

The results demonstrate a clinical scenario with $100 \%$ specificity for ovulation confirmation and provide the theoretical background for a future development of a competitive lateral flow assay for the detection of PDG in the urine. 


\section{Graphical abstract}

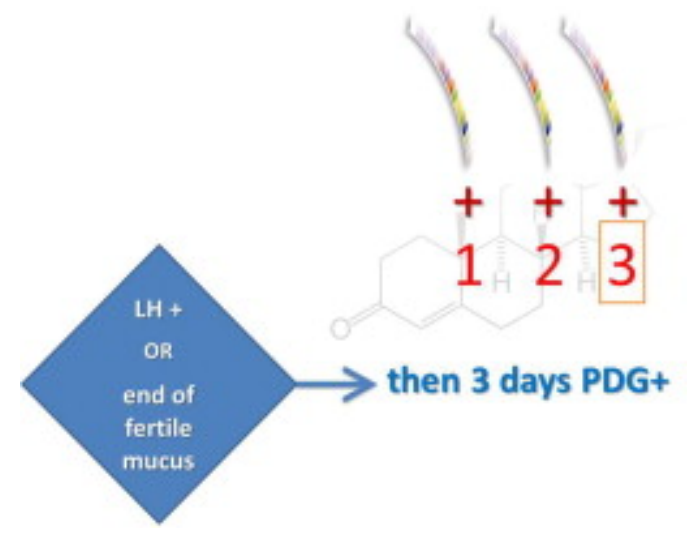

\section{Keywords}

Menstrual cycle, Pregnandiol-3-glucuronide, Luteinizing hormone, Natural family planning, Ovulation, Fertility awareness methods

\section{Introduction}

Since the mid-20th century, urinary hormone assays have been proposed to help identify the fertile phase of the menstrual cycle [1], [2]. These assays can be used by women wishing to postpone pregnancy by using Fertility Awareness Based Methods (FABM). Three urinary hormonal testing methods have long been proposed in scientific literature to help identify the ovulatory period: oestrone3-glucuronide (E1G), pregnanediol-3a-glucuronide (PDG), and luteinizing hormone (LH) ${ }^{[3]}$, [4]. In addition to urinary markers, cervical mucus is one of the most widely used biological markers for selfestimating the beginning and end of the fertile phase in a menstrual cycle $[5],[6],[7]$. Furthermore, two clinical indicators of ovulation are broadly known, the mucus peak symptom ${ }^{[6]},{ }^{[8]},{ }^{[9]},{ }^{[10]},{ }^{[11]}$ and the basal body temperature (BBT) rise. Instead of mucus or BBT as indicators, a hormonal marker of ovulation would be useful. Some home-based ovulation predictor kits based on LH identification in the urine have been marketed for this purpose $\frac{[12]}{1}, \underline{[13]}$. However, in a previous study, it was discovered that ovulation may sometimes be missed with LH kits if their threshold are above $20 \mathrm{mlU} / \mathrm{mL}$ [11]. Furthermore, there are many different amplitudes, configurations, and durations of the $\underline{\mathrm{LH}}$ surge that

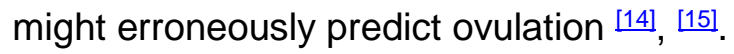

A more direct and objective measure to confirm ovulation is the urinary measure of the metabolite of post ovulatory progesterone. Several authors have suggested that the use of single morning urinary samples of PDG above a threshold would be a better indicator of ovulation [16], [17], [18]. Even more, devices using this concept were at one time considered for marketing ${ }^{[19]}$. However, this approach was vulnerable to error due to the nature of the assays of urinary PDG and the variability in PDG

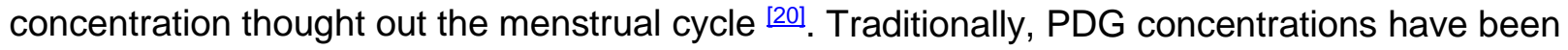
corrected for creatinine to avoid these problems; however, this correction adds a technical difficulty to 
develop simple-to-use, home-based-point of care devices. As a result, other methods combining electronic urinary monitors are being studied to address this problem [21], although they are likely to be cost prohibitive for many women. Despite the latter looking very promising, it is clear that other more affordable, easy to use, and versatile methods would be welcomed by FABM users.

The combination of robust markers of ovulation, namely urinary hormones and cervical mucus, could synergistically improve the identification of the fertile and infertile phases. In the mid-nineteen nineties, researchers collected information from normally ovulating women regarding daily urinary hormone measures, recordings of basal body temperature, cervical mucus observations, and serial ovarian ultrasound in order to study the possibility for a PDG urine hormonal assay ${ }^{[11]}$. A database of information was created but due to legal-commercial disclosure agreements, the results regarding the role of PDG in confirming post-ovulatory infertility were not published until now; this paper will present these results. In this study we assessed the potential diagnostic qualities of these markers, focusing on a given urinary PDG concentration threshold to identify the post-ovulatory infertile phase of the cycle.

\section{Experimental}

\subsection{Subjects}

This European prospective study was conducted between 1996 and 1997 in eight fertility centers: Aixen-Provence, Dijon, and Lyon (France), Milano and Verona (Italy), Düsseldorf (Germany), Liège (Belgium), and Madrid (Spain). It included healthy menstruating women aged 18-45 years with previous menstrual cycles of 24-34 days who had experience recording basal body temperature and monitoring cervical mucus. However, for the purpose of the current analysis, no women were excluded based on the duration of the cycle.

Women with a history of infertility, pelvic inflammatory disease, cycle disturbances, disturbed follicular development, or current hormone therapy were excluded from the study. We also excluded women who had had gynaecological surgery, a delivery within the last three months, women who were breastfeeding, and competitive athletes.

The study included 107 women and analyzed an average of three cycles per woman for a total of 326 cycles. The original study that collected the data ${ }^{[11]}$ was approved by the local ethics committee (Comité Consultatif de Protection des Personnes dans la Recherche Biomédicale de Lyon). All participants gave their written informed consent. The study procedures were carried out in accordance with the Ethical Standards for Human Experimentation established by the Declaration of Helsinki.

\subsection{Methods}

\subsubsection{Mucus symptoms}

Study participants checked for changes in cervical mucus two or three times daily, recording the sensation (dry, moist, wet, or slippery) and the consistency (tacky, creamy, or stretchy) of the mucus. This information allowed for the ability to distinguish between (i) days with no mucus felt or seen; (ii) days with mucus felt or seen but not having the characteristics of high fertility; and (iii) days with mucus 
that felt wet or slippery or that resembled an egg-white and had a stretchy appearance. The last day of clear, stretchy and/or lubricative mucus discharge was called the peak symptom [9], [22]

\subsubsection{Hormone assessments}

Assays were carried out on the first morning urine (FMU) with two 10-12 $\mathrm{mL}$ aliquots frozen on the day of collection at $-20^{\circ} \mathrm{C}$ in tubes containing gentamicin sulphate. On the day of analysis, the aliquots were thawed in a single laboratory and tested in duplicates for quantitative detection of oestrone-3glucuronide (E1G-ng/mL), pregnanediol-3a-glucuronide (PDG- $\mu \mathrm{g} / \mathrm{mL}$ ), follicle stimulating hormone (FSH-mIU/mL), and luteinizing hormone $(\mathrm{LH}-\mathrm{mIU} / \mathrm{mL})$ using time-resolved fluorometric immunosorbent assays (Delfia). Each hormonal sample was repeated twice: the relative difference (i.e. CV) was respectively $5.96 \%, 10.79 \%, 8.66 \%$ and $7.17 \%$ for PDG, FSH, LH and E1G. We cannot provide detailed information on assay performance except the intra-assay CV's. This data remains within the property of the funding company.

\subsubsection{Ultrasound scans}

The serial transvaginal ovarian ultrasounds (with follicle measurements) started either at the onset of the fertile cervical mucus or at the detection of the $\mathrm{LH}$ surge by the home test. These scans were carried out every other day until the largest follicle reached $16 \mathrm{~mm}$, then every day until evidence of ovulation. To note that while there is increasing evidence to indicate that multiple ovarian follicular

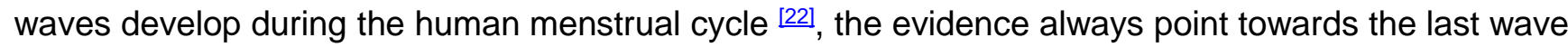
being the ovulatory single event of a given cycle ${ }^{[23]}$. The same physician at each centre performed the scans. The ultrasound-determined day of ovulation (US-DO) was the 24-h period that separated the sight of a mature follicle on one scan and either of the following on the second scan: (i) a change in the follicle size, shape, or sonographic density; (ii) follicle rupture; (iii) the presence of an early corpus luteum; (iv) the presence of free fluid in the cul-de-sac. If a woman missed an ultrasound examination, the US-DO was the first day after the last pre-ovulatory ultrasound with a follicle $\geqslant 18 \mathrm{~mm}$ or the second day with a follicle $<18 \mathrm{~mm}$.

\subsection{Measured outcomes}

\subsubsection{Fertility definitions}

The fertile phase was estimated during the pre-ovulatory phase as the period stretching from the first day of menses to the end of the US-DO. The infertile phase was defined as the day after ovulation day, up to the following menses.

Positive PDG test was defined as a test result above a defined concentration threshold. A negative $P D G$ test was defined as a test result below that threshold. Different PDG concentration thresholds in FMU samples were analyzed for specificity, sensitivity, true negative and true positive cycles. A cycle with at least one day with a positive PDG test in the fertile phase was classified as a false positive: i.e. PDG concentration was high despite being during the potentially fertile phase. A cycle with all days with negative PDG tests in the fertile phase was classified as a true negative: i.e. PDG was appropriately low during the potentially fertile phase. A cycle with days in the infertile phase with positive PDG tests 
was classified as a true positive: i.e. PDG was appropriately high during the infertile phase. A cycle without at least one day in the infertile phase with a positive PDG test was classified as a false negative: i.e. PDG was always low despite being in the infertile phase.

The sensitivity was estimated as the proportion of true positives, that is, cycles with appropriate recognition of the post-ovulatory infertile phase. The specificity was estimated as the proportion of true negatives, that is, cycles with appropriate recognition of the pre-ovulatory fertile phase. Lack of specificity creates the risk of unplanned pregnancy, and therefore, a high specificity for ovulation confirmation was the main aim of the study. Achieving a specificity of $100 \%$ would mean that there is no positive test in the absence of ultrasound-confirmed ovulation, in other words, we would not want a woman to think she was infertile if she has not yet ovulated.

\subsubsection{Tested scenarios for different PDG thresholds}

The following scenarios were tested: $(A)$ Use of the daily PDG test alone starting the first day of the cycle; (B) Use of the daily PDG test only after a first positive urine LH kit result (LH threshold of $20 \mathrm{mIU} / \mathrm{mL}$ ); and (C) Use of the PDG test only after the disappearance of highly fertile type mucus at the vulva, i.e. return to absence of mucus or mucus without the characteristics of high fertility. In the event of a second wave of highly fertile type mucus during the testing, the test was re-started following the disappearance of the second wave of highly fertile type mucus. The chosen LH threshold of

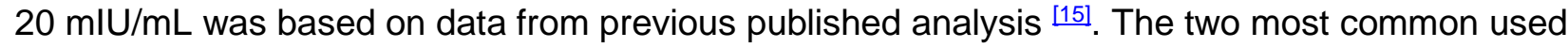
thresholds of commercially available urinary LH kits are 20 and 25 . The respective specificity and sensitivity were found to be 0.95 and 0.43 for a threshold of 20; and, 0.97 and 0.34 for 25 . Given on these results, we chose 20 to be the best as it had the higher sensitivity.

\subsubsection{PDG test models and interpretation of the results}

We postulated two models of using the PDG test based on current FABM practices: (1) One day of positivity above a certain threshold is considered to be sufficient to declare infertility; or (2) Three consecutive days of positivity are observed before infertility is declared.

\subsection{Statistical analysis}

The best threshold was obtained through secondary analysis by performing a statistical analysis in a range from 0.5 to $15 \mu \mathrm{g} / \mathrm{mL}$ and determining the threshold based on specificity and sensitivity. Sensitivity and specificity were estimated with their 95\% confidence intervals. Then a ROC (the Receiver Operating Characteristic) curve was used to describe the evolution of sensitivity and specificity according to the given threshold. A descriptive analysis of the cycle characteristics was performed using geometric mean, standard deviation, minimum, median, and maximum for quantitative data. Frequency was used for categorical variables. All statistical analyses were performed using the $R$ software version 2.13.0 (The R Foundation for Statistical Computing). A $p$-value $<0.05$ was considered for statistical significance. 


\section{Results}

Table 1 depicts the participants' characteristics including hormonal profiling. The 107 subjects studied were 19-44 years old. Sixty-nine of them (64\%) had at least one child before the study. The BMIs ranged between 17.1 and 28.3. Eleven women reported current smoking. The mean cycle length was 28.1 days (range 22-44 days). The mean time to ovulation was 14.8 days (range 9-33 days) and the mean post-ovulatory phase length was 13.3 days (range 7-17 days). In 28 cycles out of the 326 monitored, the first ultrasound was performed after ovulation and, in 15 others, it was not possible to determine exactly the day of ovulation by ultrasound. This left 283 ovulatory cycles for analysis. In a sub-analysis, out of the 206 available cycles with complete records on mucus coding, eight cycles (4\%) showed two waves of fertile-type cervical mucus separated by a vaginal dry phase. Ovulation followed the last wave. It is well recognized that this is a product of variations in hormonal patterns within the menstrual cycle ${ }^{[24]}$. In addition, as previously published ${ }^{[32]}$, no differences were observed between different BMI groups and PDG mean levels: 12.41(0.56), $13.09(0.53), 11.70(0.80) \mu \mathrm{g} / \mathrm{mL}$ for BMl ranges of $<19.2,19.2-23.4$, and $>23.4$ respectively.

Table 1. Women and cycles characteristics.

\section{Characteristics}

Women

Age (years)

Age at Menarche (years)

Body mass index $\left(\mathrm{kg} / \mathrm{m}^{2}\right)$

Sport activity (h/week)

Regular smokers (\%)

Vegans (\%)

Cycles

Cycle length (days)

Follicular phase (days)

Luteal phase (days)

FSH $(\mathrm{m} / \mathrm{U} / \mathrm{mL})$

Early follicular phase

Periovulatory phase

Luteal phase

$L H(m / U / m L)$

Early follicular phase

Periovulatory phase

Luteal phase

E1-3-G ( $\mathrm{ng} / \mathrm{mL})$

Early follicular phase

Periovulatory phase

\section{Mean ( \pm sem)}

Minimum

Maximum

$32.42(0.35)$

19

44

$13.17(0.1)$

$21.27(0.15)$

$1.16(0.13)$

9

17.12

0

28.34

9

11

4

$28.07(0.17)$

22

44

$14.76(0.17)$

9

7

33

$13.35(0.1)$

3.61 (0.19)

0.06

0.08

26.26

$5.24(0.26)$

0.06

21.42

$1.73(0.1)$

$3.56(0.13)$

0.08

11.77

$15.5(0.66)$

0.54

51.82

$6.19(0.37)$

0.08

52.99

$10.61(0.34)$

1.17

45.44

50.76 (1.87) 


\section{Characteristics}

Luteal phase

$P d-3 \alpha-G(\mu g / m L)$

Early follicular phase

Periovulatory phase

Luteal phase
Mean ( \pm sem)

$29.72(1.21)$

$2.34(0.09)$

0.18

0.31

1.53
Maximum

213.52

19.62

14.68

64.84

Average hormonal concentrations were estimated at the three phases of the cycle: at days 2,3 , and 4 of the cycle for the early follicular phase, at US-DO \pm 1 for the periovulatory phase, and at US-DO $+5,+7$, and +9 for the luteal phase.

The specificity and sensitivity of the PDG test in a range from 0.5 to $15 \mu \mathrm{g} / \mathrm{mL}$ with all various proposed scenarios is depicted in the Receiver Operating Characteristic curves in Fig. 1. The main goal of this study was to achieve a low false positive rate, that is, highest specificity, to ensure the lowest simulated pregnancy rate and to confirm ovulation. The ideal concentration threshold in a FMU sample for PDG positivity was found to be at $5 \mu \mathrm{g} / \mathrm{mL}$.

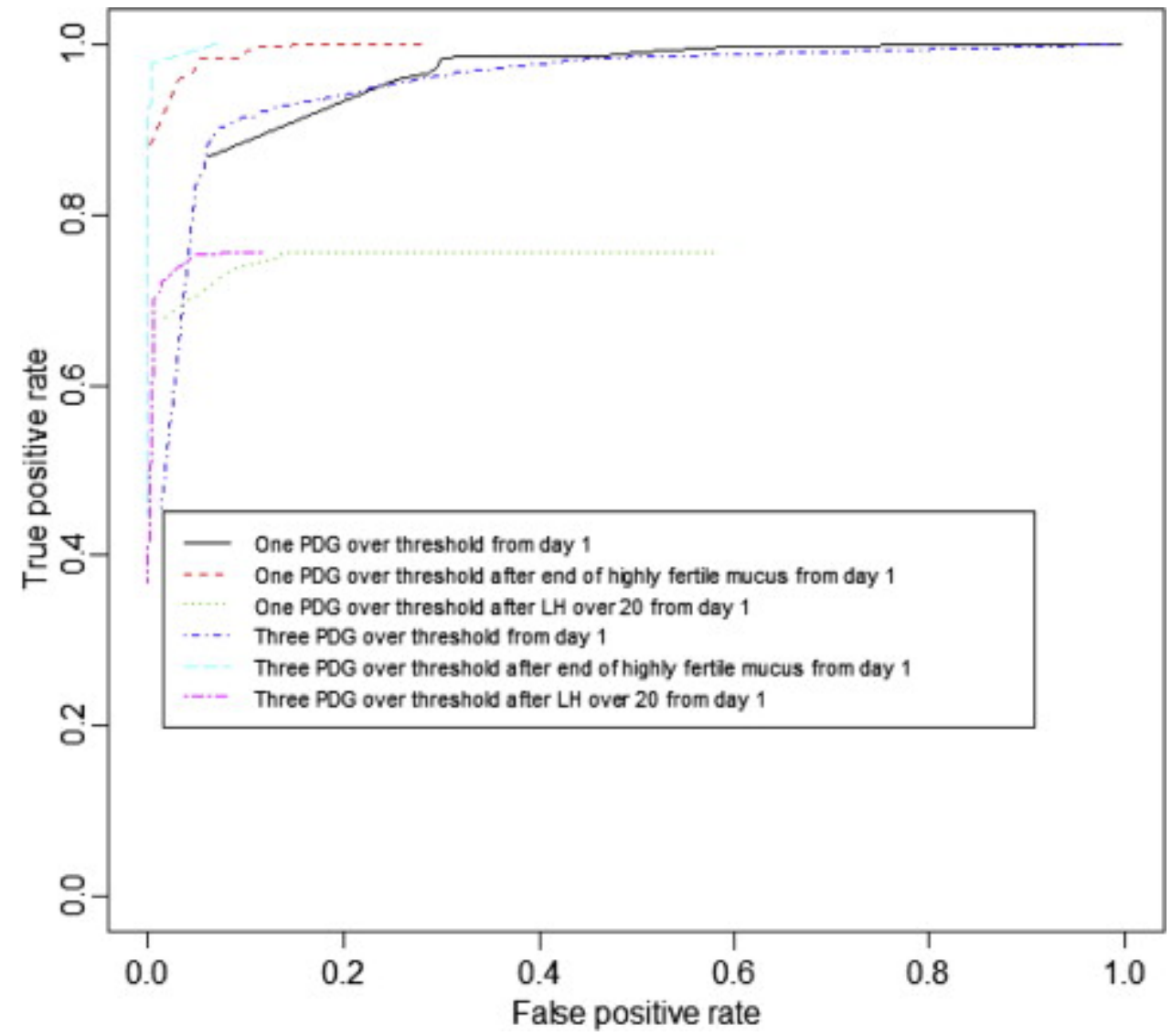

Fig. 1. ROC curves presenting the evolution of true and false positive rates according to the chosen threshold of PDG tests; one ROC curve per scenario.

The two scenarios with the highest specificity are Scenario B; using the PDG test for three consecutive days after a first positive urine LH kit result and Scenario $\mathrm{C}$ which is the most specific; using the PDG test for three consecutive days after high fertility cervical mucus is detected. 
Table 2, Table 3 show the number of daily PDG tests used for each of the three scenarios, the number of recognized true infertile days by the testing, the test's specificity and sensitivity.

Table 2. Estimated measures of the performance of PDG positivity in the one-day model (threshold: $5 \mu \mathrm{g} / \mathrm{mL}$ ).

Testing scenarios

(A) Daily PDG testing alone from day 112.1

(B) PDG daily testing only after a positive $\mathrm{LH}$ test

(C) PDG testing following peak fertility

(i.e. presence of high fertile type mucus at the vulva followed by a change to

non-fertile type)
Number of daily testing

One positive PDG result

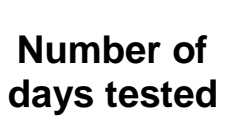

Number of recognized true infertile days $\begin{array}{cc}\text { Sensitivity } & \text { Specificity } \\ \% & \%\end{array}$

$0.986 \quad 0.534$
$0.756 \quad 0.776$
2.3
6.5

9.5

0.984

0.907

Table 3. Estimated measures of the performance of PDG positivity in the 3-day model (threshold: $5 \mu \mathrm{g} / \mathrm{mL}$ ).

\section{Testing scenarios}

(A) Daily PDG testing alone from day 1

(B) PDG daily testing only after a positive

$\mathrm{LH}$ test

(C) PDG testing following peak fertility (i.e. presence of high fertile type mucus at the vulva followed by a change to non-fertile type)

\section{Number of daily testing \\ Three daily consecutive positive PDG results}

Number of Number of days tested

18.6 recognized true infertile days

Sensitivity Specificity $\% \quad \%$ $\begin{array}{lll}6.6 & 0.929 & 0.862\end{array}$
5.2
6.1
0.721
0.986

Table 2 shows the results associated with scenarios using the one-day model of positive PDG testing above a specific threshold. Table 3 refers to the three-day model (three days of consecutive positive tests). The three scenarios were applied to both models.

The most specific scenario, which combined the use of three positive PDG tests after the identification of high fertility mucus (Scenario C), resulted in 92\% of sensitivity for ovulation. In this scenario, 6 days were tested per cycle and 7.6 days are recognized as infertile during the post-ovulatory phase. If we ignore the $8 \%$ of cycles with lack of sensitivity, 8.1 days are recognized as infertile during the postovulatory phase. 
The other scenarios were all limited in some way. First, when using LH positivity as the starting point for the PDG test (Scenario B), there was limited sensitivity with only $76 \%$ of the cycles achieving a positive $\mathrm{LH}$ test and thus precluding the use the PDG test. Second, when using the PDG test alone (Scenario A), there was limited specificity and the PDG test occasionally read positive before ovulation had taken place.

In a sub-analysis to take into account sport activity, data was available for 76 women. We did it to assess whether sport activity may be associated with a reduced luteal PDG. First, we divided participants in three groups: no sport-activity, intensive sport-activity for less than $3 \mathrm{~h}$ per week and for more than $3 \mathrm{~h}$ per week. The sport activity was assessed only once per woman and we analyzed the data accordingly. Within each group, PDG values from three days of each cycle were averaged to obtain one value per cycle (i.e. the mean PDG level of days US-DO +5, +7 , and +9). We then averaged all these mean PDG levels for all the cycles within a given group. In this analysis, the results obtained were $11.23,12.01$ and 11.14 for the three groups respectively $(p=0.77$, i.e. the difference was not statistically significant).

\section{Discussion}

The present study predicts that the use of three consecutive days of PDG testing over a threshold of $5 \mu \mathrm{g} / \mathrm{mL}$ in a FMU sample will result in a perfect specificity for ovulation confirmation following fertilitytype mucus identification. The $100 \%$ confirmation would allow women to identify the absolute infertile period after ovulation for the sake of avoiding pregnancy. On the other hand, the rationale behind the fact that the combination of LH/PDG did not give a perfect specificity can be found on recent LH research ${ }^{[15]}$. It is now known that $\mathrm{LH}$ levels present some variability in amplitude, duration and configuration with ovulation sometimes occurring later than one day after the $\underline{\mathrm{LH}}$ surge. It is then possible that our proposed PDG test protocol may give a false negative since it may test too early to show a PDG surge. However, we believe the high specificity obtained is still within the clinically relevant range $(S p=0.986)$.

The beginning of the fertile phase still requires the use of a first indicator; either, a count of days from the onset of the cycle, or the presence of mucus, felt or seen, at the vulva ${ }^{[6]}$ or the detection of a significant rise in urinary E1G, either alone or in various combinations $\stackrel{[25]}{[26]}, \underline{[27]}$. To identify the end of the fertile phase, our results confirm the interest of using either a LH test first or to rely on days following the presence of cervical mucus for FABM users, and then confirm the infertility using three consecutive days of PDG testing over a threshold of $5 \mu \mathrm{g} / \mathrm{mL}$ in a FMU sample. A noon sample of urinary LH above a set threshold has been considered sufficient to identify the imminence of ovulation [28]. Alternatively, instead of relying on the same threshold for every woman, electronic devices have been developed to identify a significant increase over a given woman's baseline hormonal levels during the previous days ${ }^{[29]}$. Our results show that three consecutive days of PDG positivity above a specific threshold following LH positivity or the end of fertile-type cervical mucus will confirm infertility with a high predictive specificity.

Clinical an-ovulatory scenarios such as pre-menopause and polycystic ovarian disease (PCOD), has been mentioned as challenges for FABM use. Firstly, the effect of these scenarios should be negligible 
in the interpretation of the PDG method; since, it is only with those ovulatory cycles that the rise of urinary PDG occurs. However, it is been long reported that there are clinical scenarios when luteal progesterone levels, and consequently PDG levels as well, may be found at lower level than normal: in women with unexplained infertility, after ovulation induction with clomiphene citrate or gonadotropins, in women with hyperprolactinemia, recurrent miscarriages, luteinizing unruptured follicles, in oligomenorrheic obese women with or without hirsutism such as PCOD, and in perimenopausal women [30], [31], [32]. Our current proposed protocol lacks the power to discriminate for all these conditions because it is based on one single threshold, yet, it could be the source of future research on the relationship between cervical mucus, LH and PDG surges. Secondly and even more importantly, it is the combination of two markers (cervical mucus or LH) plus PDG that may provide the safety net needed in these conditions with reduced PDG level. For instance, it is recognized that use of cervical mucus monitoring in FABM can help to identify approaching ovulation even in pre-menopause ${ }^{\text {[24]. }}$. Thirdly, in clinical situations such as vaginal infections when the cervical mucus essentially becomes non-interpretable, the use of urinary LH testing could be used as substitute as mentioned in our protocols. Finally, despite the fact that the clinical question of accurately identifying the infertile phase of the menstrual cycle is an old one (1, 2, and 20), the proposed model is new. No quantitative assessment of the use of PDG as adjunct to the concurrent use of FABM has ever been published, nor a study has used such a large dataset.

With these results, the next major challenge will be the development of a simple competitive lateral flow assay for the detection of PDG in the urine. The ability to develop such assays has been available for several years and the manufacturing processes of these tests are well known. Similar urinary tests such as those used to identify commonly abused substances could be adapted for this purpose given that PDG and these substances have comparable molecular weights $\stackrel{\text { [33] }}{\text {. }}$

One further theoretical limitation of our study is that the algorithms used were based on multiple cycles per participants, leading to potentially to overestimates of specificity and sensitivity. To quantify for this effect, a mixed regression model for repeated measurement was used to describe the PDG level during the luteal phase (at US-DO $+5,+7$, and +9 ). The inter-women and intra-women coefficients of variation were respectively $30 \%$ and $11 \%$. However, we did not use a validation dataset to confirm the sensitivity and specificity: our estimates of performance can be overly optimistic. It would be wise to confirm these results using other datasets in future research. This fact might indeed contribute to an overestimate of specificity, but not necessarily invalidate the results in a clinical setting. Likewise, the fact that our PDG assays were tested only among European women, it may potentially limits the study's results yet not necessarily invalidating our findings ${ }^{[34]}$. A clinical study based on the proposed PDG devices would again need to address concerns such a different population and racial differences.

Given that this test has a dual purpose (to confirm the end of the fertile period for women wishing to get pregnant and identifying the infertile phase for those wishing to avoid pregnancy) it is very versatile. The 100\% specificity for ovulation in these scenarios would be helpful to identify those women with adequate ovulation for the purpose of an infertility work-up, providing a home-based alternative for serum progesterone testing. For those wishing to postpone pregnancy, the high specificity for ovulation demonstrated here provides a simple and very reliable identification for the post-ovulatory infertile phase. In order to make this approach practical, the cost of the simple PDG test ought to remain low. 
Future studies could assess the two best scenarios (B and C) for women who are seeking to avoid and achieve pregnancy.

On a side note, it was noted that in $2 \%$ of the cycles (6/283), menses occurred beyond the generally accepted 16 days post-ovulation, in these few cycles, we question whether the ultrasound determination of the day of ovulation was off by one day or two or an early pregnancy loss occurred. Our assessment of these rare events led us to conclude that the potential bias would not be significant.

In order to provide women with a simple, home-based test to identify the absolute infertile period after ovulation, we have demonstrated a new objective measure that is $99-100 \%$ specific for the ovulation event as confirmed by ultrasound. In order to avoid the previous challenge of the individual woman's menstrual cyclic variations in urinary concentrations of PDG, we have proposed a novel model that employs three consecutive days of PDG tests above a threshold of $5 \mu \mathrm{g} / \mathrm{mL}$ after either LH positivity or highly fertile mucus. This model is $99-100 \%$ specific for ovulation depending of the scenario used and is thus a very promising tool for women wanting a conservative and reliable measure to complement their Fertility Awareness Based Method.

\section{Potential conflict of interest}

H.B. is employed for a company that specializes on the development of competitive lateral flow assays.

\section{Acknowledgments}

The authors thank everybody who provided feedback of this manuscript including Drs. Victoria Jennings, Sophie Dubus, Anne Leduy, Isabelle Ecochard, Marie Grisard Capelle, Enriqueta Barranco, Michele Barbato, Sandro Girotto Robin MacLaren, and Marion Gimmler from the natural family planning clinics as well as all the women who took part in this study. We thank Jean Iwaz, Ph.D., scientific advisor, for suggestions and criticisms of the manuscript.

This study was partially supported by Quidel Corporation, San Diego, CA. USA.

\section{References}

1 J.B. Brown. The relationship between urinary oestrogens and oestrogens produced in the body, J Endocrinol, 16 (1957), pp. 202-212

2 W.P. Collins, P.O. Collins, M.J. Kilpatrick, P.A. Manning, J.M. Pike, J.P. Tyler. The concentrations of urinary oestrone-3-glucuronide, LH and pregnanediol-3alpha-glucuronide as indices of ovarian function, Acta Endocrinol (Copenh), 90 (2) (1979 Feb), pp. 336-348

3 K.A. O'Connor, E. Brindle, R.C. Miller, J.B. Shofer, R.J. Ferrell, N.A. Klein, M.R. Soules, D.J. Holman, P.K. Mansfield, J.W. Wood. Ovulation detection methods for urinary hormones: precision, daily and intermittent sampling and a combined hierarchical method, Hum Reprod, 21 (6) (2006 Jun), pp. 1442-1452 
4 World Health Organization. Temporal relationships between ovulation and defined changes in the concentration of plasma estradiol-17B, LH, FSH and P. I. Probit analysis, Am J Obstet Gynecol, 138 (1980), pp. 383-390

5 J.L. Bigelow, D.B. Dunson, J.B. Stanford, et al. Mucus observations in the fertile window: a better predictor of conception than timing of intercourse, Hum Reprod, 19 (2004), pp. 889-892

6 E.L. Billings, J.J. Billings, J.B. Brown. Symptoms and hormonal changes accompanying ovulation, Lancet, 1 (1972), pp. 282-284

7 J.B. Stanford, K.R. Smith, D.B. Dunson. Vulvar mucus observations and the probability of pregnancy, Obstet Gynecol, 101 (2003), pp. 1285-1292

$8 \mathrm{~V}$. Jennings, I. Sinai. Further analysis of the theoretical effectiveness of the TwoDay method of family planning, Contraception, 64 (3) (2001 Sep), pp. 149-153

9 T.W. Hilgers, G.E. Abraham, D. Cavanaugh. Natural family planning. I. The peak symptom and estimated time of ovulation, Obstet Gynecol, 52 (1978), pp. 575-582

10 R. Fehring. Accuracy of the peak day of cervical mucus as a biological marker of fertility, Contraception, 66 (2002), pp. 231-235

11 R. Ecochard, H. Boehringer, M. Rabilloud, H. Marret. Chronological aspects of ultrasonic, hormonal, and other indirect indices of ovulation, BJOG, 108 (8) (2001 Aug), pp. 822-829

12 M.S. Nielsen, S.D. Barton, H.H. Hatasaka, J.B. Stanford. Comparison of several one-step home urinary luteinizing hormone detection test kits to OvuQuick, Fertil Steril, 76 (2) (2001 Aug), pp. 384-387

13 P.R. Brezina, E. Haberl, E. Wallach. At home testing: optimizing management for the infertility physician, Fertil Steril, 95 (6) (2011 May), pp. 1867-1878

14 S.J. Park, L. Goldsmith, J. Skurnick, A. Wojtczuk, G. Weiss. Characteristics of the urinary luteinizing hormone surge in young ovulatory women, Fertil Steril, 88 (3) (2007), pp. 684690

15 A. Direito, S. Bailly, A. Mariani, R. Ecochard. Relationships between the luteinizing hormone surge and other characteristics of the menstrual cycle in normally ovulating women, Fertil Steril, 99 (1) (2013 Jan), pp. 279-285

16 M.V. Sauer, R.J. Paulson. Utility and predictive value of a rapid measurement of urinary pregnanediol glucuronide by enzyme immunoassay in an infertility practice, Fertil Steril, 56 (5) (1991 Nov), pp. 823-826

17 J.H. Denari, Z. Farinati, P.R. Casas, A. Oliva. Determination of ovarian function using first morning urine steroid assays, Obstet Gynecol, 58 (1) (1981 Jul), pp. 5-9

18 H. Adlercreutz, J. Brown, W. Collins, U. Goebelsman, A. Kellie, H. Campbell, J. Spieler, G. Braissand. The measurement of urinary steroid glucuronides as indices of the fertile period in women. World Health Organization, task force on methods for the determination of the fertile period, special programme of research, development and research training in human reproduction, J Steroid Biochem, 17 (6) (1982 Dec), pp. 695-702

19 Moffat S. Taking the natural option: As couples search for satisfactory forms of contraception, some 'rediscover' a traditional method; others are introduced to birth control by an unconventional messenger. Los Angeles Times. 1993 Jan 7. < http://articles.latimes.com/1993-01-07/news/vw9351 birth-control-methods> [accessed online 01.04.13]. 
20 M.V. Sauer, R.J. Paulson, P. Chenette, J. Frederick, F.Z. Stanczyk. Effect of hydration on random levels of urinary pregnanediol glucuronide, Gynecol Endocrinol, 4 (3) (1990 Sep), pp. 145149

21 L.F. Blackwell, P. Vigil, B. Gross, C. d'Arcangues, D.G. Cooke, J.B. Brown. Monitoring of ovarian activity by measurement of urinary excretion rates of estrone glucuronide and pregnanediol glucuronide using the Ovarian Monitor, Part II: reliability of home testing, Hum Reprod, 27 (2) (2012 Feb), pp. 550-557

22 A.R. Baerwald, G.P. Adams, R.A. Pierson. Ovarian antral folliculogenesis during the human menstrual cycle: a review, Hum Reprod Update, 18 (1) (2012 Jan-Feb), pp. 73-91

23 A.R. Baerwald, G.P. Adams, R.A. Pierson. A new model for ovarian follicular development during the human menstrual cycle, Fertil Steril, 80 (1) (2003 Jul), pp. 116-122

24 J.B. Brown. Types of ovarian activity in women and their significance: the continuum (a reinterpretation of early findings), Hum Reprod Update, 17 (2) (2011 Mar-Apr), pp. 141-158

25 J. Bonner, A. Flynn, G. Freundl, R. Kirkman, R. Royston, R. Snowden. Personal hormone monitoring for contraception, Br J Family Planning, 24 (1999), pp. 124-128

$26 \mathrm{~K}$. May. Home monitoring with the ClearPlan easy fertility monitor for fertility awareness, $\mathrm{J}$ Int Med Res, 29 (Suppl. 1) (2001), pp. 14A-20A

27 G. Freundl, E. Godehardt, P.A. Kern, P. Frank-Herrmann, H.J. Koubenec, Ch. Gnoth. Estimated maximum failure rates of cycle monitors using daily conception probabilities in the menstrual cycle, Hum Reprod, 18 (12) (2003 Dec), pp. 2628-3331

28 A.F. Khattab, F.A. Mustafa, P.J. Taylor. The use of urine LH detection kits to time intrauterine insemination with donor sperm, Hum Reprod, 20 (9) (2005 Sep), pp. 2542-2545

29 T.P. Bouchard, S.J. Genuis. Personal fertility monitors for contraception, CMAJ, 183 (1) (2011 Jan 11), pp. 73-76

30 P. Petsos, H. Mamtora, W.A. Ratcliffe, D.C. Anderson. Inadequate luteal phase usually indicates ovulatory dysfunction: observations from serial hormone and ultrasound monitoring of 115 cycles, Gynecol Endocrinol, 1 (1) (1987 Mar), pp. 37-45

31 M.P. Hamilton, R. Fleming, J.R. Coutts, M.C. MacNaughton, C.R. Whitfield. Luteal phase deficiency: ultrasonic and biochemical insights into pathogenesis, $\mathrm{Br} \mathrm{J}$ Obstet Gynaecol, 97 (7) (1990 Jul), pp. 569-575

32 K.A. O'Connor, R. Ferrell, E. Brindle, B. Trumble, J. Shofer, D.J. Holman, M. Weinstein. Progesterone and ovulation across stages of the transition to menopause, Menopause, 16 (6) (2009 Nov-Dec), pp. 1178-1187

33 C.R. Wong, H.Y. Tse (Eds.), Lateral flow immunoassay, Human Press, New York, NY (2009), p. 133

34 K.A. O'Connor, E. Brindle, D.J. Holman, N.A. Klein, M.R. Soules, K.L. Campbell, F. Kohen, C.J. Munro, J.B. Shofer, B.L. Lasley, J.W. Wood. Urinary estrone conjugate and pregnanediol 3glucuronide enzyme immunoassays for population research, Clin Chem, 49 (7) (2003 Jul), pp. $1139-1148$ 